\title{
Developing an Integrated Model to Enhance the Efficiency in the Detection and Erasing of Duplicate Files from Cloud $^{1}$
}

\author{
Rishit Garkhel
}

DOI: 10.37648/ijrst.v10i04.005

Received: 19 $9^{\text {th }}$ August, 2020; Accepted: 04 ${ }^{\text {th }}$ October, 2020; Published: $29^{\text {th }}$ October, 2020

\begin{abstract}
Identifying and disposing of the copied document is one of the serious issues in the wide space of information cleaning and information quality in the framework. Ordinarily, a similar sensible true element might have numerous portrayals in the information distribution centre. Copy disposal is hard because it is brought about by a few blunders like typographical mistakes and various pictures of similar consistent worth. Our primary aim of this study is to recognise specific and inaccurate representations by utilising copy description and end rules. This methodology is used to work on the proficiency of the information. The significance of information precision and quality has expanded with the blast of information size. In the copy disposal step, just one duplicate of accurate copied records or documents is held and dispensed with other copy records or documents. The end cycle is vital to delivering cleaning information. Before the end sequence, the similitude limit esteems are determined for every one of the records available in the informational collection. The closeness limit admires significant for the end communication.
\end{abstract}

\section{INTRODUCTION}

Duplicate record recognition is the method of recognising unique or different records that allude to one special true substance or item if their similarity surpasses specifically removed esteem. Nonetheless, the records comprise of other fields, making the copy recognition issue significantly more chaotic. A standard-based methodology is proposed for the copy location issue. This standard is created with the additional limitation to acquire the great consequence of the guidelines. These levels define the circumstances and patterns for similar records. A general if, else rule is utilised in this exploration work for copy information recognisable proof and copy information end. Regularly copy information disposal proceeds as the last advance, and this progression needs to occur while coordinating two sources or performed on a generally incorporated basis. The blend of characteristics can be utilised to recognise copy records. The copy disposal must hold just one best duplicate of the copy record, and the leftover copy records ought to be removed. Correct duplicate records are distinguished utilising the conviction factor and the limit esteem. Copy information is disposed of depending on the number of unavailable abilities, the scope of every field esteem, information nature of each field worth, and data description. Copy records are distinguished by utilising expressive and high segregation power ascribes. As a general rule, copy records can have countless missing fields. Subsequently, entries could be disposed of depending on the quantity of misplaced qualities in each copy record. Copy record is disposed of if the copy record has more missing qualities than other copy records.

\footnotetext{
${ }^{1}$ How to cite the article: Garkhel R., Developing an Integrated Model to Enhance the Efficiency in the Detection and Erasing of Duplicate Files from Cloud, IJRST, Oct-Dec 2020, Vol 10, Issue 4, 38-42, DOI: http://doi.org/10.37648/ijrst.v10i04.005
} 


\section{EXECUTION}

Certain devices are vital to the preparation of advanced pictures. These incorporate numerical devices like convolution, Fourier examination, factual portrayals, and manipulative devices, such as connected codes and track codes. We will introduce these devices with no particular inspiration.

Convolution

A few potential documentations show the convolution of two (multidimensional) signs to deliver a yield signal. The most widely recognised are:

$\mathrm{c}=\mathrm{a} \otimes \mathrm{b}=\mathrm{a}^{*} \mathrm{~b}$

In 2D ceaseless space:

$$
c(x, y)=a(x, y) \otimes b(x, y)=\int_{-\infty-\infty}^{+\infty} \int_{-\infty}^{+\infty} a(\chi, \zeta) b(x-\chi, y-\zeta) d \gamma d \zeta
$$

In 2D discrete space:

$$
c[m, n]=a[m, n] \otimes b[m, n]=\sum_{j=-\infty}^{+\infty} \sum_{k=-\infty}^{+\infty} a[j, k] b[m-j, n-k]
$$

\section{Fourier Transforms}

It delivers one more portrayal of a sign, explicitly portraying a weighted number of complicated exponentials. Due to Euler's equation:

$$
e^{j q}=\cos (q)+j \sin (q)
$$

Since we can say that $21 \mathrm{j}=-$ of Fourier transform represents a signal of (2D) as sines and cosines weighted sum. The formula and definition of forward and inverse Fourier transform is presented above. Taking an image of I and its Fourier transformation $A$, the forward change goes from spatial to frequency, remaining unchanged (whether the Fourier is continuous or discrete).

Histogram-Based Operations

A significant class of point activities depends on the control of a picture histogram or a locale histogram.
Frequently, a picture is filtered so that the subsequent brilliance esteems don't utilise the accessible unique reach. Could recognize this in the brightness value of the histogram by extending the histogram to the limit of the range available. We are trying to improve this condition. When comparing from two or more images on a particular reason, like construction, traditionally, it is normalized to the standard histogram. This can be particularly helpful when the pictures have been gained under various conditions. The most widely recognised histogram standardisation strategy is histogram balance. One endeavour is to change the histogram using a capacity $\mathrm{b}=f(\mathrm{a})$ to be consistent for all brilliance esteems. This would relate to a splendour conveyance where all qualities are similarly likely. Lamentably, for a subjective picture, one can estimate this outcome.

\section{Techniques}

The suggested System detects duplicate records: The information cleaning and normalisation measure are utilised because true data sets contain consistently filthy, boisterous, inadequate, and erroneously designed data. The entire undertaking of information cleaning and normalisation measure is changing the crude info information into the clear cut, reliable structures, just as the goal of irregularities in how data is addressed. An electronic copy record discovery structure is planned and carried out in this exploration to beat the missing elements and capacities in the now accessible systems. The proposed structure gives discovery online copy record recognition administration with no requirement for extra setups or establishments on the customer side machine.

Information Cleaning and Standardisation: -

The currently utilised methods that perform cleaning and normalisation don't cover all spaces of typographical varieties. In this manner, the information cleaning and normalisation measure rely upon the introduced language augmentations. During this cycle, information is brought together, standardised and normalised. This means working on the nature of the in-stream information and making the info tantamount and more usable. It improves acknowledging names and identifying their language, 
which is a significant stage for perceiving typographic variations. The steps of pre-processing are done for normalization of character level, parsing and splitting of characters, transforming the connected names into an official format using searching.

\section{Language Extensions: -}

For non-English dialects, normalising names through character standardisation is more troublesome and includes a few stages. These means are characterised as administrations from base to top, where high-level assistance can rely upon a lower administration and call it. For instance, the name breakdown technique relies on the knowledge of prefix and post of name in the training set.

Parsing of full name and conversion in canonical form

Names should be parsed and converted into official forms with regards to prefixes and postfixes. For instance, with a normal word splitter parser, a complete name like "Slam Laxman hanuman" or "Marco Ram Mohan " are parted by the parser into three words and shows up as though it comprises three titles. The Arabic language augmentation and the Dutch language expansion characterise standard structure prudent name parsing measure. This interaction utilises the pre-put away prefixes table to revamp "Shyam Rahman" as a solitary first name and "Smash Mohan" as a compound surname. The last advance here is the bringing together interaction which binds together the variations of "Shyam Rahman" including "Bashful El Rahman", "Shyam Rahman", "Timid Al Rahman" to a solitary brought together sanctioned structure. In the favourable to the presented System, the SME can make a standard structure to address input information that coordinates with few situations such all (shyl\%) will be supplanted by (shyl\%).

Breaking down and Reorganization

When the string contains name fields in the full name, the name is broken down into separate strings like first, middle, and last. For instance, Narendra Damodardas Modi, into Narendra, damodardas modi. Few languages and application like French and English consider writing the last name first and the first name last, whereas Arabic consider first name appears the first. Changing the request for the names addressed in a language to coordinate with the transcribed names in another dialect is a significant stage for adjusting the names. The principal cycle includes forestalling the revealing of copies, and the quantity of copy reports in open-source bug storehouses recommends that this cycle isn't adequate. The subsequent interaction includes distinguishing documents as a message is being triaged. A bug triage ordinarily endeavours this ID by scrutinising the task's most often detailed bugs list and by performing a look at the reports in the archive.

\section{Ordering/Blocking: -}

Each copy record discovery issue is related to some issue area ordering/impeding conditions. These conditions recognise which record sets are potential up-and-comers as indicated by their similitude in specific fields. Likewise, field coordinating is an obstructing plan that limits the number of record sets to be looked at later. Ordering/hindering is liable for decreasing the quantity of produced sets of records by forestalling the examination of record matches that will surely cause a bogus outcome.

Removal from multilevel data algorithm for duplicate rules: -

A staggered dataset has a verifiable scientific categorisation or idea tree, similar to the model displayed in Figure. However, the things in the dataset that exist at the most minimal idea level are essential for a progressive design and association. In this way, for instance, 'ME' is a thing at the most minimal level of the scientific classification; however, it also has a place with the general idea class of 'Science' and the more advanced class 'Engg'. Each section in the order has one paternal (or prompt, super subject) with a way back to the root conceivable from any place in the progression.

Proposed Algorithm with Recursive Stringcoordinating with Proposed Algorithm with adjusted iterative Word-Similar Algorithm is utilised for inexact string coordinating. The assessment is performed by going the edge esteem from 0.45 to 0.65 with the hole of 0.05 . While running DCS++, acquired the most reliable outcomes at 0.65 . 


\section{CONCLUSION}

In this examination work, a structure is intended to clean copy information to develop information quality and help any subject situated information. In this exploration work, a productive copy location and end approach is created to acquire great aftereffects of copy recognition and disposal by decreasing bogus up-sides. Duplication and information linkage are significant undertakings in the pre-processing venture for some information discovering projects. Further, develop information quality before the information is stacked into enormous information records. Finding estimated copies in a huge information distribution centre is important information for the executives and assumes a basic part in the information cleaning measure. Analysing this work produced a result that shows that our proposed approach consumes less time and yield greater accuracy. The System is mostly evolved to speed up the copy information discovery and end measure and to build the nature of the information by recognising genuine copies and adequately severe to keep out bogus up-sides. The exactness and proficiency of copy end procedures are improved by presenting the idea of a conviction factor for a standard. Information purifying is a mind-boggling and testing issue. This standard-based technique assists with dealing with the intricacy yet doesn't eliminate that intricacy.

\section{REFERENCES}

[1]. Radu-Ioan,Ciobanu,Valentin Cristea, Ciprian Dobre and Florin Pop, Big Data Platforms for the Internet of Things,2014,Springer

[2]. Flavio Bonomi, Rodolfo Milito, Preethi Natarajan and Jiang Zhu,Fog Computing: A Platform for Internet of Things and Analytics, springer (2014)

[3]. Shintaro Yamamoto, Shinsuke Matsumoto,Sachio Saiki, and Masahide Nakamura Kobe University, 1-1 Rokkodai-cho, Nada-ku, Kobe, Hyogo 657-8501, Japan, Using Materialized View as a Service of Scallop4SC for Smart City Application Services (2014)

[4]. Mukherjee, A.; Datta, J.; Jorapur, R.; Singhvi, R.; Haloi, S.; Akram, W. "Shared disk big data analytics with Apache Hadoop" (18-22 Dec. 2012)

[5]. Kudakwashe Zvarevashe1, Dr. A Vinaya Babu, Towards MapReduce Performance Optimization: A Look into the Optimization Techniques in Apache Hadoop for Bigdata Analytics (2014)

[6]. Gartner: Hype cycle for big data, 2012. Technical report (2012)

[7]. IBM, Zikopoulos, P., Eaton, C.: Understanding Big Data: Analytics for Enterprise Class Hadoop and Streaming Data. 1st edn. McGraw-Hill Osborne Media, New York (2011)

[8]. Schroeck, M., Shockley, R., Smart, J., Romero-Morales, D., Tufano, P.: Analytics: The real-world use of big data. IBM Institute for Business Value - executive report, IBM Institute for Business Value (2012)

[9]. Evans, D.: The internet of things - how the next evolution of the internet is changing everything. Technical report (2011)

[10]. Cattell, R.: Scalable sql and nosql data stores. Technical report (2012)

[11]. Apache: Hadoop (2014) (Online 20 Oct 2015)

[12]. Jo Foley, M.: Microsoft drops dryad; puts its big-data bets on hadoop. Technical report (2011)

[13]. Locatelli, O.: Extending nosql to handle relations in a scalable way models and evaluation framework (2012012)

[14]. Robinson, I., Webber, J., Eifrem, E.: Graph Databases. O'Reilly Media, Incorporated (2013) 
[15]. DeCandia, G., Hastorun, D., Jampani, M., Kakulapati, G., Lakshman, A., Pilchin, A., Sivasubramanian, S., Vosshall, P., Vogels,W.: Dynamo: amazon's highly available key-value store. SIGOPS Oper. Syst. Rev. 41, 205220 (2007) Big Data Management Systems for the Exploitation 89

[16]. Riak: Riak (Online Oct 2015)

[17]. Apache: Couchdb (Online; Oct 2015)

[18]. MongoDB: Mongodb (Online; Oct 2015)

[19]. Hypertable: Hypertable (Online; Oct 2015)

[20]. Rabl, T., Gómez-Villamor, S., Sadoghi, M., Muntés-Mulero, V., Jacobsen, H.A., Mankovskii, S.: Solving big data challenges for enterprise application performance management. Proc. VLDB Endow. 5, 1724-1735 (2012)

[21]. Neo Technology, I.: Neo4j, the world's leading graph database. (Online;Oct 2015)

[22]. Amato, A., DiMartino, B., Venticinque, S.: Semantically augmented exploitation of pervasive environments by intelligent agents. In: ISPA, pp. 807-814.(2012)

[23]. Jing Zhang, “A Distributed Cache for Hadoop File Distribution system in Real time Cloud Services”, 2012 ACM/IEEE 13th International Conference on Grid Computing.

[24]. Pig.apachi.org (online Oct 2015). 